\title{
The position of the germinal vesicle and the chromatin organization together provide a marker of the developmental competence of mouse antral oocytes
}

\author{
Michele Bellone ${ }^{1}$, Maurizio Zuccotti ${ }^{2}$, Carlo Alberto Redi ${ }^{3}$ and Silvia Garagna ${ }^{1,4}$ \\ ${ }^{1}$ Dipartimento di Biologia Animale, Università degli Studi di Pavia, Piazza Botta 9-10, 27100 Pavia, Italy, ${ }^{2}$ Sezione di \\ Istologia e Embriologia, Dipartimento di Medicina Sperimentale, Università degli Studi di Parma, Via Volturno 39, \\ 43100 Parma, Italy, ${ }^{3}$ Fondazione IRCCS, Policlinico San Matteo, Viale Golgi 19, 27100 Pavia, Italy and ${ }^{4}$ Centro di \\ Ricerca Interdipartimentale di Ingegneria Tissutale e Centro di Eccellenza in Biologia Applicata, Università degli Studi \\ di Pavia, Via Ferrata 1, 27100 Pavia, Italy
}

Correspondence should be addressed to M Zuccotti; Email: maurizio.zuccotti@unipv.it;

S Garagna at Dipartimento di Biologia Animale, Università degli Studi di Pavia; Email: silvia.garagna@unipv.it

\begin{abstract}
Based on their chromatin organization, antral oocytes can be classified into two classes, namely surrounded nucleolus (SN, chromatin forms a ring around the nucleolus), and not surrounded nucleolus (NSN, chromatin has a diffuse pattern). Oocytes of both classes are capable of meiotic resumption, but while SN oocytes, following fertilization, develop to term, NSN oocytes never develop beyond the two-cell stage. A recent study has shown that the position of the germinal vesicle (GV) can be used as a morphological marker predictive of oocyte meiotic competence, i.e. oocytes with a central GV have a higher meiotic competence than oocytes with an eccentric GV. In the present study, we have associated both markers with the aim of identifying, with more accuracy, the oocytes' developmental competence. Following their isolation, antral oocytes were classified on the basis of both SN and NSN chromatin configuration and their GV position, matured to metaphase II and fertilized in vitro. We demonstrated that the position of the GV is a good marker to predict the oocytes' developmental competence, but only when associated with the observation of the chromatin organization.

Reproduction (2009) 138 639-643
\end{abstract}

\section{Introduction}

A number of morphological and molecular markers have been suggested to predict the meiotic and developmental competence of antral oocytes (Albertini et al. 2003, Wang \& Sun 2007). One of these parameters is the chromatin organization of the germinal vesicle (GV), according to which antral oocytes can be classified into two classes named surrounded nucleolus (SN), in which the chromatin forms a ring around the nucleolus, and not surrounded nucleolus (NSN), in which the chromatin has a more diffuse pattern (Parfenov et al. 1989, Mattson \& Albertini 1990, Debey et al. 1993, Zuccotti et al. 1995). In addition to a different chromatin configuration, other differences have been described between mouse $\mathrm{SN}$ and NSN oocytes. For example, the heterochromatin surrounding the nucleolus in mouse $\mathrm{SN}$ oocytes is mainly AT-rich satellite-DNA of centromeric origin and involves most of the centromeres, whereas in NSN oocytes only the centromeres carrying the nucleolar organizing regions are associated with the nucleolus (Longo et al. 2003). Microtubule organizing centers form around the nucleus of SN oocytes, but not around that of NSN oocytes (Wickramasinghe \& Albertini 1992, Can et al. 2003). In addition, the nucleoli of NSN oocytes are vacuolated and less compact than those of SN oocytes (Debey et al. 1993). These morphological differences have biological relevance as they have been correlated with changes in transcription (Moore et al. 1974, Kaplan et al. 1982, Bouniol-Baly et al. 1999, Christians et al. 1999, Liu \& Aoki 2002, Miyara et al. 2003). NSN oocytes remain transcriptionally active and synthesize all classes of RNA, whereas $\mathrm{SN}$ oocytes are transcriptionally inactive (Debey et al. 1993, Bouniol-Baly et al. 1999). Another difference between the two types of oocytes is the extent of DNA methylation, histone acetylation, and histone methylation, which is higher in SN oocytes (Kageyama et al. 2007). Oocytes possessing NSN and SN chromatin organizations have been found in a variety of mammals, including rats (Mandl 1962), monkeys (Lefevre et al. 1989), pigs (Crozet et al. 1981), and humans (Parfenov et al. 1989).

Oocytes of both classes are capable of meiotic resumption (Zuccotti et al. 1998, 2002, 2008, Inoue et al. 2008), but while SN oocytes, following 
fertilization, reach the blastocyst stage and develop to term, NSN oocytes are developmentally incompetent and never develop beyond the two-cell stage (Zuccotti et al. 1998, 2002, Inoue et al. 2008).

In a recent study, Brunet \& Maro (2007) have shown that the position of the GV in mouse antral oocytes is a morphological feature predictive of the oocyte's meiotic competence: according to these authors, oocytes with a central GV (CGV) have a higher meiotic competence than those with an eccentric GV (EGV). The authors proposed the use of this marker to evaluate the oocyte's quality. Although in the majority of the mammals studied, such as primates, ungulates and most of the rodents, the position of the GV is eccentric, the rat and mouse are exceptions to this tendency (Albertini \& Barrett 2004).

In the present study we have analyzed both parameters together, with the aim of defining, with more accuracy, those mouse oocytes that are developmentally competent. Following isolation from the ovarian surface, oocytes were classified on the basis of SN or NSN chromatin configuration and the position of their GV. Each group of oocytes was matured to the metaphase II (MII) stage, fertilized in vitro and observed throughout preimplantation development.

\section{Results}

\section{Developmentally competent SN oocytes have a lower frequency of CGVs compared to that of developmentally incompetent NSN oocytes}

Following the classification based on their $\mathrm{SN}$ - or NSN-type of chromatin configuration (Fig. 1), antral oocytes were separated into three groups depending on the position of their GV ( $\rho$ value; Fig. 2 ). In the large majority $(91.2 \%)$ of NSN oocytes the GV occupied a central position (Table 1, 3-isobutyl-1-methylxanthine $\left.\left(\mathrm{BMX}^{-}\right)\right)$; a small percentage showed either an intermediate $(4.4 \%)$ or a peripheral $(4.4 \%)$ GV position. Compared to NSN, SN oocytes had a lower frequency of central $(64.5 \%)$ and higher frequencies of both intermediate $(17.3 \%)$ and peripheral (18.2\%) GVs $(P<0.001)$.
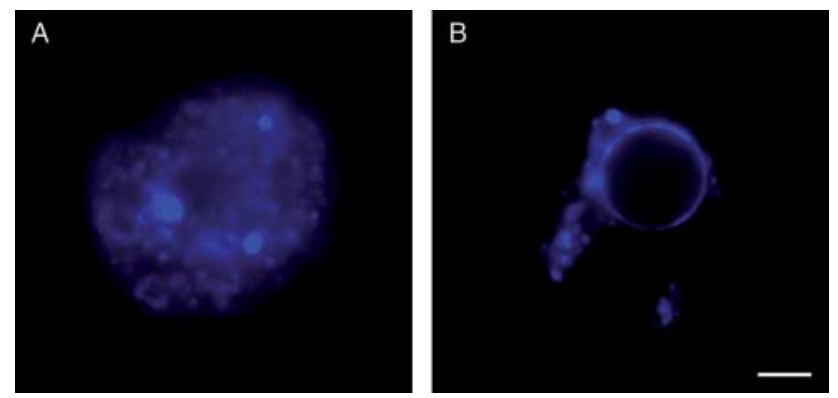

Figure 1 (A) NSN antral oocyte and (B) SN antral oocyte. Bar $=2 \mu \mathrm{m}$.
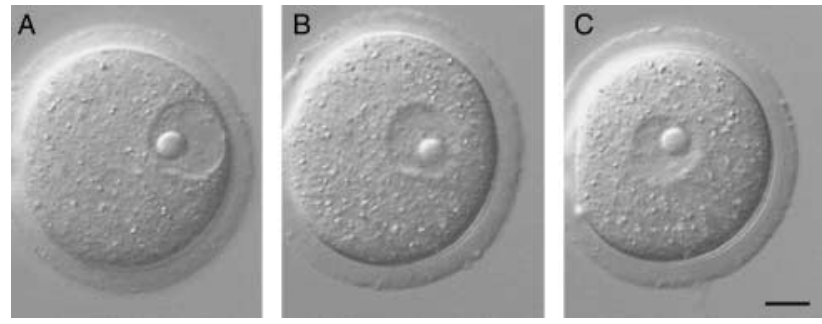

Figure 2 Antral oocytes classified on the basis of their GV position ( $\rho$ value): (A) peripheral $(\rho<0.66)$; (B) intermediate $(0.66<\rho<0.33)$, and $(C)$ central $(\rho>0.33)$. Bar $=15 \mu \mathrm{m}$.

To verify if the culture conditions during the time interval between the isolation of the oocytes and the acquisition of the images $(\sim 1 \mathrm{~h})$ could change the position of the GV, we added $0.2 \mathrm{mM}$ IBMX to the M2 medium during the entire handling period. IBMX is a nonspecific inhibitor of phosphodiesterases that prevents meiotic resumption by promoting the persistence of the perinuclear microtubule network (Alexandre et al. 1989) and by delaying the progression of nuclear maturation, without affecting cytoplasmic maturation (Barretto et al. 2007). Following the addition of IBMX to the medium, we observed a significant increase in the frequencies of central and intermediate GVs and a significant decrease in peripheral GVs $(P<0.05$; Table 1$)$ of $\mathrm{SN}$ oocytes compared to the frequencies observed without IBMX. On the contrary, in NSN oocytes, the variation in central, intermediate and peripheral GV frequencies was not significant $(P=0.272)$.

\section{SN oocytes with a CGV are more developmentally competent compared to those with an EGV}

In order to determine the relationship between chromatin organization, GV position and developmental competence, we separately fertilized MII-derived SN $\left(\mathrm{MIIN}^{\mathrm{SN}}\right)$ and MII-derived NSN (MII ${ }^{\mathrm{NSN}}$ ) oocytes with a CGV or an EGV (the sum of intermediate

Table 1 Frequencies of central, intermediate, and peripheral germinal vesicles (GVs) in antral not surrounded nucleolus (NSN) and surrounded nucleolus ( $\mathrm{SN}$ ) oocytes isolated and classified in the absence (3-isobutyl-1-methylxanthine $\left.\left(\mathrm{IBMX}^{-}\right)\right)$or presence $\left(\mathrm{IBMX}^{+}\right)$ of IBMX.

\begin{tabular}{lccc}
\hline & \multicolumn{3}{c}{ GV position $(\%)$} \\
\cline { 2 - 4 } $\begin{array}{l}\text { Chromatin } \\
\text { configuration }\end{array}$ & Central & Intermediate & Peripheral \\
\hline NSN & & & \\
IBMX ${ }^{-}(n=68)^{+}$ & 91.2 & 4.4 & 4.4 \\
IBMX ${ }^{+}(n=58)$ & 82.8 & 12 & 5.2 \\
SN & & & \\
IBMX ${ }^{-}(n=110)^{*}{ }^{+}$ & 64.5 & 17.3 & 18.2 \\
IBMX $^{+}(n=131)^{*}$ & 75.6 & 20.6 & 3.8 \\
\hline$\chi^{2}$ test: $^{*} P<0.05,{ }^{+} P<0.001$. &
\end{tabular}


Table 2 Developmental rate of antral oocytes classified on the basis of the eccentric (EGV) or central (CGV) position of their germinal vesicle (GV).

\begin{tabular}{|c|c|c|c|c|c|c|}
\hline \multirow{2}{*}{$\begin{array}{l}\text { Oocytes } \\
\text { classification }\end{array}$} & \multirow[b]{2}{*}{$\mathbf{G V}(n)$} & \multicolumn{5}{|c|}{ Stage of development $(\% \pm$ S.D. $)$} \\
\hline & & MII & One-cell & Two-cell & Four-cell & Blastocyst \\
\hline \multicolumn{7}{|l|}{ SN } \\
\hline CGV & 163 & $74.8 \pm 6.2$ & $52.9 \pm 16.5$ & $91.6 \pm 11.1$ & $39.5 \pm 6.4$ & $19 \pm 3.3^{*}$ \\
\hline EGV & 72 & $71.5 \pm 16.1$ & $58.3 \pm 5.7$ & $76.3 \pm 20.6$ & $19.8 \pm 16.9$ & $3.1 \pm 6.3^{*}$ \\
\hline \multicolumn{7}{|l|}{ NSN } \\
\hline CGV & 81 & $23.4 \pm 7.4$ & $50 \pm 22$ & $54.2 \pm 41.7$ & 0 & 0 \\
\hline EGV & 18 & $18.8 \pm 23.9$ & $25 \pm 50$ & $12.5 \pm 25$ & 0 & 0 \\
\hline
\end{tabular}

All values are expressed as the mean \pm s.D. of four separate experiments. Pre-implantation development has been calculated considering one-cell embryos as $100 \%$ (Student's $t$-test: ${ }^{*} P<0.05$ ).

and peripheral GVs; this shortened the time of oocytes classification.) As shown in Table 2, $\mathrm{MII}^{\mathrm{SN}}$-CGV oocytes displayed a higher rate of development to the blastocyst stage when compared to MII ${ }^{\mathrm{SN}}$-EGV oocytes $(P<0.05)$. Interestingly, CGV oocytes have the same maturation rate to MII of EGV oocytes both in the $\mathrm{SN}(P=0.717)$ and NSN $(P=0.722)$ groups. Confirming previous results (Zuccotti et al. 1998, 2002, Inoue et al. 2008), in vitro fertilized MII ${ }^{\mathrm{NSN}}$ oocytes cease their development at the two-cell stage.

\section{Discussion}

A major problem in the use of assisted reproductive technologies is the difficulty to make predictions on the quality of the available oocytes. Using animal models, several attempts have been made to develop methods that can be used to predict an oocyte's developmental competence. Clearly, whatever is the method, a prerequisite is that it must be based on a type of analysis that does not damage the oocyte. A simple observation of the female gamete at the light microscope may be the easiest and least damaging solution. To this end, in a recent study, Brunet \& Maro (2007) have shown that the position of the GV within mouse antral oocyte correlates with the female gamete meiotic competence, i.e. the more central the nucleus, the better the antral oocyte's capability to resume meiosis.

In the present study, we combined the position of the GV and the chromatin organization to identify those oocytes with a better developmental competence. We found that oocytes with a CGV are more abundant in developmentally incompetent NSN (91.2\%) when compared to developmentally competent SN (64.5\%) oocytes. When antral oocytes, previously classified on the basis of both chromatin organization and position of their GVs, were cultured in vitro to MII, we did not find differences in the maturation rate between CGV and EGV oocytes, differently from what it has been previously reported (Brunet \& Maro 2007). Perhaps, these different findings might be explained with the strain of mice used in the two studies (outbred OF1, Brunet \& Maro 2007; inbred B6C3F1, this study), but this point needs to be investigated further.

Despite a similar maturation rate, following IVF MII $^{\mathrm{SN}}$-CGV oocytes developed to blastocyst with a higher frequency compared to $\mathrm{MII}^{\mathrm{SN}}$-EGV oocytes. This observation indicates that the central position of the GV per se is not a sufficient marker of oocytes with a high developmental competence. In fact, if antral oocytes were selected only on the basis of their CGV, NSN oocytes also could be picked up, thus decreasing the blastocyst rate of an IVF experiment.

In the majority of mammals examined, the position of the GV is eccentric during the oocyte's growth; at the antral stage, whilst in most species it remains eccentric, in the rat and mouse the GV assumes a central position (for a review see Albertini \& Barrett 2004) and only after the assembly of the meiotic spindle, the chromosomes migrate back to the oocyte's cortex (Longo \& Chen 1985, Verlhac et al. 2000, Leader et al. 2002, Li et al. 2008). Consistent with these observations was our finding that the GV of antral mouse oocytes is predominantly located centrally. It is noteworthy that when oocytes are cultured in the presence of IBMX (used to inhibit meiotic resumption), the frequency of eccentric $\mathrm{SN}$ oocytes results significantly lower and that of CGVs is higher compared to culture conditions in the absence of IBMX. These results suggest that the position of the GV may change during the time spent between isolation and classification, depending on the experimental conditions, as previously suggested (Sanfins et al. 2003, Albertini \& Barrett 2004).

In summary, our results show that the combined analysis of chromatin organization and GV position is a valuable tool to predict mouse oocytes' developmental competence. Although this method of oocytes' classification has limited applicability for clinical purposes, both for the toxicity of the Hoechst dye and the necessity to preserve the cumulus-oocyte integrity while maturing human oocytes in vitro, it might find applications when studying the oocytes' developmental competence in those species in which the presence of NSN and SN oocytes has been described. 


\section{Materials and Methods}

\section{Animals}

Five to eight week-old B6C3F1 female mice and 16-20 week-old B6C3F1 male mice were purchased from Charles River Laboratories (Calco, Italy) and maintained according to the Guide for Care and Use of Laboratory Animals. The animals were killed by cervical dislocation; every effort was made to minimize the number of animals used and their suffering and the ethics committee approved the procedures involving the use of animals.

\section{Chemical reagents}

All reagents were purchased from Sigma Co., unless otherwise stated.

\section{Oocytes isolation and classification}

Females were primed with 3.5 IU Folligon (Intervet srl, Milan, Italy) $48 \mathrm{~h}$ before the isolation of the ovary. The ovarian surface was punctured with a thin sterile glass needle to release antral oocytes in M2 medium (Millipore, Billerica, MA, USA). To visualize their chromatin organization, oocytes were first freed from the surrounding cumulus cells by gentle pipetting through a mouth-controlled micropipette, transferred into a $20 \mu \mathrm{l}$ droplet of M2 medium containing $50 \mathrm{ng} / \mathrm{ml}$ Hoechst 33342 and incubated at $37^{\circ} \mathrm{C}$ for $10 \mathrm{~min}$. Single oocytes were transferred into $4 \mu \mathrm{l}$ droplets of $\mathrm{M} 2$ medium and observed with a AX70 microscope (Olympus, Ibaraki, Japan) at $\times 100$ magnification for not more than 2-3 s under u.v. fluorescence light (excitation $350 \mathrm{~nm}$, emission $461 \mathrm{~nm}$ ) and classify on the basis of their SN or NSN chromatin organization (Fig. 1a and b; Zuccotti et al. 1995). After isolation and classification, using a holding micromanipulation pipette mounted on an inverted microscope equipped with DIC optics (IX70, Olympus), each oocyte was carefully positioned in order to visualize the GV at its most peripheral position (Brunet \& Maro 2007) and pictures were taken using a CCD camera (FY-K58; JVC Italia spa, Segrate, Italy).

\section{In vitro maturation of antral oocytes}

Following isolation and classification, antral oocytes were rinsed in $\alpha$-MEM medium containing 5\% FBS (Invitrogen), $2 \mathrm{mM}$ L-glutamine (Invitrogen), $5 \mathrm{mM}$ Taurine, and $26 \mu \mathrm{g} / \mathrm{ml}$ sodium pyruvate and finally transferred into drops of $\alpha$-MEM for in vitro maturation. Oocytes were incubated at $37^{\circ} \mathrm{C}$ for $15 \mathrm{~h}$ under an atmosphere of $5 \% \mathrm{CO}_{2}, 5 \% \mathrm{O}_{2}$, and $90 \% \mathrm{~N}_{2}$. Following maturation, only those oocytes that had progressed through to the MII stage were transferred to a drop of Whittingham medium (Wt; Whittingham 1971) and fertilized.

\section{IVF of antral oocytes}

Sperm were isolated as previously described (Zuccotti et al. 1998) and incubated for $60 \mathrm{~min}$ in $100 \mu \mathrm{l}$ drops of Wt medium at a final concentration of $1.5 \times 10^{6} \mathrm{sperm} / \mathrm{ml}$. Oocytes at the
MIl phase, obtained following in vitro maturation of SN or NSN antral oocytes, were transferred into the insemination drop and incubated at $37{ }^{\circ} \mathrm{C}$ under $5 \% \mathrm{CO}_{2}$ in air. Based on the presence of a second polar body, as a sign of fertilization, presumptive one-cell stage embryos were pooled, transferred from $\mathrm{Wt}$ medium to a drop of M16 medium (Fulton \& Whittingham 1978 , Scott \& Whittingham 1996) under an atmosphere of $5 \%$ $\mathrm{CO}_{2}, 5 \% \mathrm{O}_{2}, 90 \% \mathrm{~N}_{2}$ and covered with mineral oil for further development.

\section{Image analysis}

Boundaries of each oocyte and its nucleus were drawn using Microsoft Power Point (Microsoft Inc). The resulting images were analyzed using ImageJ freeware software (NIH, http:// rsbweb.nih.gov/ij/). Centroid and Feret's radius were measured for each oocyte and its corresponding GV. The $\rho$ value (Brunet \& Maro 2007) was used to define the position of the GV within the oocyte according to this formula:

$\rho=\frac{d\left(C_{\mathrm{GV}}-C_{\mathrm{OO}}\right)}{\left(R_{\mathrm{OO}}-R_{\mathrm{GV}}\right)}$

were $d\left(C_{\mathrm{GV}}-C_{\mathrm{OO}}\right)$ is the distance between the GV and the oocyte centroid and $R_{\mathrm{GV}}$ and $R_{\mathrm{OO}}$ are the GV and the oocyte Feret's radius respectively. Depending on their $\rho$ values, oocytes were separated into three groups: $\rho<0.33$, oocytes with a CGV; $0.33<\rho<0.66$, oocytes with a GV in an intermediate position; $\rho>0.66$, oocytes with a peripheral GV (Fig. 2).

\section{Statistical analysis}

Experiments were replicated at last three times. Relative frequencies of maturation and development were compared using the Student's $t$-test. The frequencies of central, intermediate and peripheral GVs between different groups were compared using the $\chi^{2}$ test. Differences were considered significant when $P<0.05$. All tests were performed with the statistical software SigmaStat 3.5 (Aspire Software International, Ashburn, VA, USA).

\section{Declaration of interest}

The authors declare that there is no conflict of interest that would prejudice the impartiality of this scientific work.

\section{Funding}

Funding for this study was provided by: FIRB 2005 (Project N. RBIP06FH7J), Fondazione CARIPLO 2006, UNIPV-Regione Lombardia Project on Material Science and Biomedicine, Fondazione I.R.C.C.S. Policlinico San Matteo, Millipore.

\section{Acknowledgements}

The authors would like to thank M Zanoni and C Vasco for their assistance in the micromanipulation of oocytes. 


\section{References}

Albertini DF \& Barrett SL 2004 The developmental origins of mammalian oocyte polarity. Seminars in Cell and Developmental Biology 15 599-606.

Albertini DF, Sanfins A \& Combelles CMH 2003 Origins and manifestations of oocyte maturation competence. Reproductive Biomedicine Online 6 35-40.

Alexandre H, Van Cauwenberge A \& Mulnard J 1989 Involvement of microtubules and microfilaments in the control of the nuclear movement during maturation of mouse oocyte. Developmental Biology 136 311-320.

Barretto LSS, Caiado Castro VSD, Garcia JM \& Mingoti GZ 2007 Role of roscovitine and IBMX on kinetics of nuclear and cytoplasmatic maturation of bovine oocytes in vitro. Animal Reproduction Science 99 202-207.

Bouniol-Baly C, Hamraoui L, Guibert J, Beaujean N, Szollosi MS \& Debey P 1999 Differential transcriptional activity associated to chromatin configuration in fully grown germinal vesicle mouse oocytes. Biology of Reproduction 60 580-587.

Brunet S \& Maro B 2007 Germinal vesicle position and meiotic maturation in mouse oocyte. Reproduction 133 1069-1072.

Can A, Semiz O \& Cinar O 2003 Centrosome and microtubule dynamics during early stages of meiosis in mouse oocytes. Molecular Human Reproduction 9 749-756.

Christians E, Boiani M, Garagna S, Dessy C, Redi CA, Renard JP \& Zuccotti M 1999 Gene expression and chromatin organization during mouse oocyte growth. Developmental Biology 207 76-85.

Crozet N, Motlik J \& Szollosi D 1981 Nucleolar fine structure and RNA synthesis in porcine oocytes during early stages of antrum formation. Biology of the Cell 41 35-42.

Debey P, Szollozi MS, Szollosi D, Vautier D, Girouse A \& Besombes D 1993 Competent mouse oocytes isolated from antral follicles exhibit different chromatin organization and follow different maturation dynamics. Molecular Reproduction and Development 36 59-74.

Fulton BP \& Whittingham DG 1978 Activation of mammalian oocytes by intracellular injection of calcium. Nature 273 149-151.

Inoue A, Nakajima R, Nagata M \& Aoki F 2008 Contribution of the oocyte nucleus and cytoplasm to the determination of meiotic and developmental competence in mice. Human Reproduction 23 1377-1384.

Kageyama S, Liu H, Kaneko N, Ooga M, Nagata M \& Aoki F 2007 Alterations in epigenetic modifications during oocyte growth in mice. Reproduction 133 85-94.

Kaplan G, Abreu SL \& Bachvarova R 1982 rRNA accumulation and protein synthetic patterns in growing mouse oocytes. Journal of Experimental Zoology 220 361-370.

Leader B, Lim H, Harrington A, Ecsedy J, Pellman D, Maas R \& Leder P 2002 Formin-2, polyploidy, hypofertility and positioning of the meiotic spindle in mouse oocytes. Nature Cell Biology 4 921-928.

Lefevre B, Gougeon A, Nome F \& Testart J 1989 In vivo changes in oocyte germinal vesicle related to follicular quality and size at midfollicular phase during stimulated cycles in the cynomolgus monkey. Reproduction, Nutrition, Development 29 523-532.

Li H, Guo F, Rubinstein B \& Li R 2008 Actin-driven chromosomal motility leads to symmetry breaking in mammalian meiotic oocytes. Nature Cell Biology 10 1301-1308.

Liu H \& Aoki F 2002 Transcription activity associated with meiotic competence in fully grown mouse GV oocytes. Zygote 10 327-332.

Longo FJ \& Chen DY 1985 Development of cortical polarity in mouse eggs: involvement of the meiotic apparatus. Developmental Biology 107 382-394.
Longo FJ, Garagna S, Merico V, Orlandini G, Gatti R, Scandroglio R, Redi CA \& Zuccotti M 2003 Nuclear localization of NORs and centromeres in mouse oocytes during folliculogenesis. Molecular Reproduction and Development 66 279-290.

Mandl AM 1962 Preovulatory changes in the oocyte of the adult rat. Proceedings of the Royal Society of London (Biology) 158 105-118.

Mattson DA \& Albertini DF 1990 Oogenesis: chromatin and microtubule dynamics during meiotic prophase. Molecular Reproduction and Development 25 374-383.

Miyara F, Migne C, Dumont-Hassan M, Le Meur A, Cohen-Bacrie P, Aubriot FX, Glissant A, Nathan C, Douard S, Stanovici A et al. 2003 Chromatin configuration and transcriptional control in human and mouse oocytes. Molecular Reproduction and Development 64 458-470.

Moore GPM, Lintern-Moore S, Peters H \& Faber M 1974 RNA synthesis in the mouse oocyte. Journal of Cell Biology 60 416-422.

Parfenov V, Potchukalina G, Dudina L, Kostyuchek D \& Gruzova M 1989 Human antral follicles: oocyte nucleus and the karyosphere formation (electron microscopic and autoradiographic data). Gamete Research 22 219-231.

Sanfins A, Lee GY, Plancha CE, Overstrom E \& Albertini DF 2003 Distinctions in meiotic spindle structure and assembly during in vitro and in vivo maturation of mouse oocytes. Biology of Reproduction 69 2059-2067.

Scott L \& Whittingham DG 1996 Influence of the genetic background and media components on the development of mouse embryos in vitro. Molecular Reproduction and Development 43 36-46.

Verlhac MH, Lefebvre C, Guillaud P, Rassinier P \& Maro B 2000 Asymmetric division in mouse oocytes: with or without Mos. Current Biology $101303-1306$.

Wang Q \& Sun QY 2007 Evaluation of oocyte quality: morphological, cellular and molecular predictors. Reproduction, Fertility, and Development 19 1-12.

Whittingham DG 1971 Culture of mouse ova. Journal of Reproduction and Fertility 14 7-21.

Wickramasinghe D \& Albertini DF 1992 Centrosome phosphorylation and the developmental expression of meiotic competence in mouse oocytes. Developmental Biology 152 62-74.

Zuccotti M, Piccinelli A, Giorgi Rossi P, Garagna S \& Redi CA 1995 Chromatin organization during mouse oocyte growth. Molecular Reproduction and Development 41 479-485.

Zuccotti M, Giorgi Rossi P, Martinez A, Garagna S, Forabosco A \& Redi CA 1998 Meiotic and developmental competence of mouse antral oocytes. Biology of Reproduction 58 700-704.

Zuccotti M, Ponce RH, Boiani M, Guizzardi S, Govoni P, Scandroglio R, Garagna S \& Redi CA 2002 The analysis of chromatin organization allows selection of mouse antral oocytes competent for development to blastocyst. Zygote 10 73-78.

Zuccotti M, Merico V, Sacchi L, Bellone M, Brink TC, Bellazzi R, Stefanelli M, Redi CA, Garagna S \& Adjaye J 2008 Maternal Oct-4 is a potential key regulator of the developmental competence of mouse oocytes. BMC Developmental Biology 897.

Received 4 June 2009

First decision 6 July 2009

Revised manuscript received 10 July 2009

Accepted 24 July 2009 